\title{
FLUORESCENCE OF A PHOTOSENSITIZER BASED ON AN INDOTRICARBOCYANINE DYE IN PHOTOCHEMOTHERAPY
}

\author{
M. P. Samtsov, ${ }^{a *}$ E. S. Voropay, ${ }^{\mathrm{b}}$ L. S. Liashenka, \\ D. G. Melnikau, ${ }^{\text {b }}$ K. N. Kapleusky, ${ }^{b}$ and A. P. Lugovskij ${ }^{a}$
}

UDC 535.37+535.34:541.14

\begin{abstract}
We present the results for studies of the spectral luminescence properties of a symmetric indotricarbocyanine dye (PDI) in HeLa tumor cells and animal tissues in vivo during a photochemotherapy session and after the end of the session. We have established that when the dye is exposed to light in tumor tissues, changes occur in the position and half-width of the dye fluorescence spectra, while in a culture of HeLa cells its spectral characteristics are constant. Based on analysis of the effect of overlap between the absorption spectra of endogenous biomolecules and the fluorescence spectra of the dye plus comparison of the experimental data with numerical modeling results, we have concluded that the observed changes in the fluorescence spectra of PD1 in vivo are due to a change in the ratio of the different forms of hemoglobin in the tumor tissue. We have shown that the spectral characteristics of PDI, fluorescing in the near IR range, correlate with the depth of tumor tissue necrosis achieved on exposure to light. We have established that tumor tissue necrosis occurs down to a depth of $2 \mathrm{~cm}$ in the case of all strains studied: $S-45, S M-1$, and $W-256$, where as a result of exposure to light, we observe an increase in the half-width and a short-wavelength shift of the fluorescence spectrum of the dye PDI, and also the intensity of its fluorescence does not recover.
\end{abstract}

Keywords: indotricarbocyanine dye, spectral properties, in vivo, hemoglobin, tissue oxygenation, photochemotherapy.

Introduction. Cancer photochemotherapy (PCT) is based on photochemical reactions with efficacy determined by the photosensitizer and depending on the light flux parameters and other light exposure conditions [1, 2]. Thus for photosensitizers for which the photoactivity mechanism is due to generation of singlet oxygen, when using light with high power density the efficiency of damage may be reduced due to decreased oxygenation of the tumor tissue [2-4]. Identification of the optimal conditions and optical radiation dose during PCT sessions is a timely problem having considerable practical importance. The possibility of on-the-fly correction of the light exposure parameters when monitoring the details of the response to light exposure may improve the treatment efficacy. A change in the optical properties of the biological tissues or the photosensitizer as a result of PCT seems likely, which can be detected by spectral methods. In connection with the fact that in the near IR there is a transparency window for biological tissue [5] and the penetration depth of light into tissues is greatest, this spectral range is the most promising for obtaining such information.

In this paper, we present the results of studies to identify any correlations between the spectral characteristics of the dye (fluorescing in the near IR range) localized in tumor cells and the efficiency of damage to tumor tissues during PCT. We have analyzed data for a culture of HeLa cells and animal tumor tissues in vivo.

Objects of Investigation and Study Procedures. As the object of investigation, we used the symmetric indotricarbocyanine dye PD1: 2-\{7-[3-methyl-3-ethyl-1-trimethylenecarbo-(6-O-D-glucosyl)-2(1H)-indolenylidene]-4-chloro3,5-(O-phenyleno)-1,3,5-hexatrien-1-yl -3-methyl-3-ethyl-1-trimethylenecarbo-(6-O-D-glucosyl)indolenylium bromide,

*To whom correspondence should be addressed.

${ }^{a}$ A. N. Sevchenko Institute of Applied Physical Problems, Belorussian State University, 7 Kurchatov Str., Minsk, 220064; e-mail: samtsov@bsu.by; ${ }^{b}$ Belorussian State University, Minsk; e-mail: voropay@bsu.by. Translated from Zhurnal Prikladnoi Spektroskopii, Vol. 78, No. 1, pp. 121-127, January-February, 2011. Original article submitted September 21, 2010. 

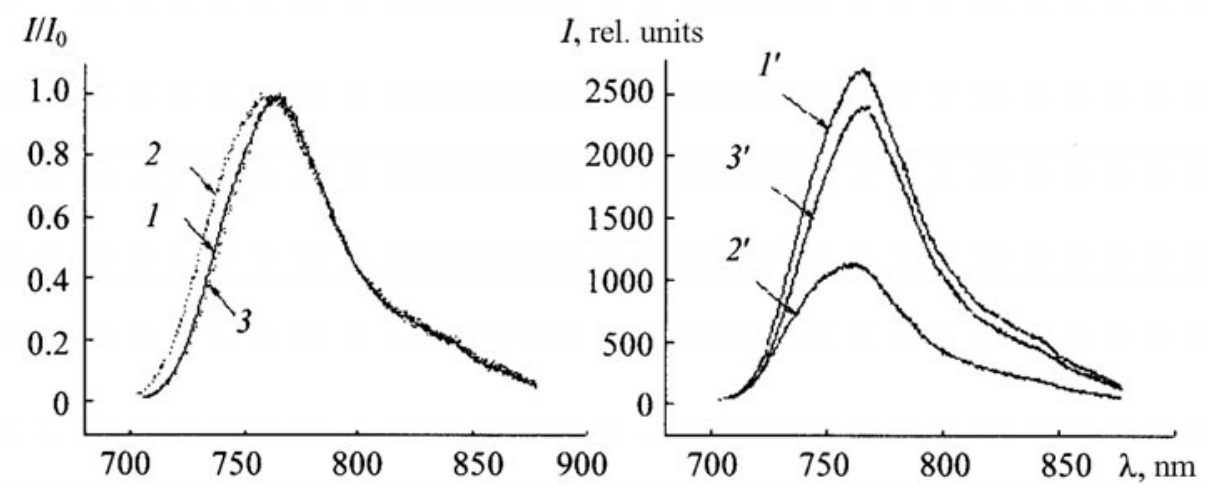

Fig. 1. Fluorescence spectra of the dye PD1 in vivo in a rat tumor before irradiation $\left(1,1^{\prime}\right)$; after exposure to light with power density $200 \mathrm{~mW} / \mathrm{cm}^{2}$ and exposure energy dose $280 \mathrm{~J} / \mathrm{cm}^{2}\left(2,2^{\prime}\right)$; in the unirradiated part of the tumor $\left(3,3^{\prime}\right)$.

which was synthesized in the spectroscopy laboratory of the A. N. Sevchenko Scientific Research Institute of Applied Physical Problems [6].

In vivo experiments were conducted on mongrel white rats with tumors of the following strains grafted in the thigh region: sarcoma 45 (S-45), sarcoma M1 (SM-1), Walker carcinosarcoma (W-256). For the investigations, groups of 6-8 animals were selected. The drug was injected intravenously (1-5 mg per $\mathrm{kg}$ weight of the animal). Exposure to light was carried out using the emission from a semiconductor laser with $\lambda=740 \mathrm{~nm}$; the area of the section of irradiated tumor was $\sim 1 \mathrm{~cm}^{2}$. The depth and extent of tumor necrosis resulting from the PCT session were determined based on the vital staining method. The spectral properties of the dye in vitro were studied in a culture of HeLa tumor cells.

The fluorescence spectra of the dye in vivo were recorded using a spectrometer developed at the A. N. Sevchenko Scientific Research Institute of Applied Physical Problems [7]. A semiconductor laser with $\lambda=683 \mathrm{~nm}$ was used in the spectrometer as the excitation source. The exciting radiation was delivered to the sample and the light emitted as fluorescence was collected by means of an optical fiber.

Results and Discussion. We studied the spectral luminescence properties of PD1 in vivo in normal and tumor tissues in rats during a PCT session. In order to determine the time over which the dye concentration in the tumor tissue is unchanged, we studied the kinetics of accumulation of the dye in the tissues after the dye was intravenously injected into the animal. The dye content in the tumor tissue in vivo was determined from the intensity of fluorescence of PD1. Based on the pharmacokinetics data in vivo for each tumor strain, we chose the optimal time for beginning exposure to light. During the selected time interval, the intensity, shape, and position of the dye fluorescence spectra did not change in the healthy and tumor tissues.

In the PCT experiments, the dye PD1 was injected in a concentration of $5 \mathrm{mg}$ per $\mathrm{kg}$ weight of the animals. Light exposure was carried out using the emission from a semiconductor laser with $\lambda=740 \mathrm{~nm}$ for power densities of the incident radiation equal to 200,300 , and $600 \mathrm{~mW} / \mathrm{cm}^{2}$ and the same exposure energy dose of $280 \mathrm{~J} / \mathrm{cm}^{2}$. Such a combination of optical radiation parameters was selected to ensure the same number of incident photons from the laser radiation for each sample.

We found that at the end of the light exposure using radiation with power density $200 \mathrm{~mW} / \mathrm{cm}^{2}$, appreciable changes are observed in the fluorescence spectrum of the dye in the tumor tissues: the half-width increased by 4-7 $\mathrm{nm}$ and the maximum was shifted by 3-4 nm toward shorter wavelengths (Fig. 1, curve 2). In this case, in the irradiated part of the tumor, there was a significant decrease in the intensity of fluorescence from the photosensitizer (curve $2^{\prime}$ ). Observation for 2 hours after the end of light exposure showed that for the irradiated section of the tumor, the intensity and shape of the dye fluorescence spectrum remained unchanged (curve 2'). For sections of the tumor which were not exposed to light, the fluorescence spectra of the dye remained the same as before beginning exposure to light (curves $1^{\prime}$ and $3^{\prime}$ ). 


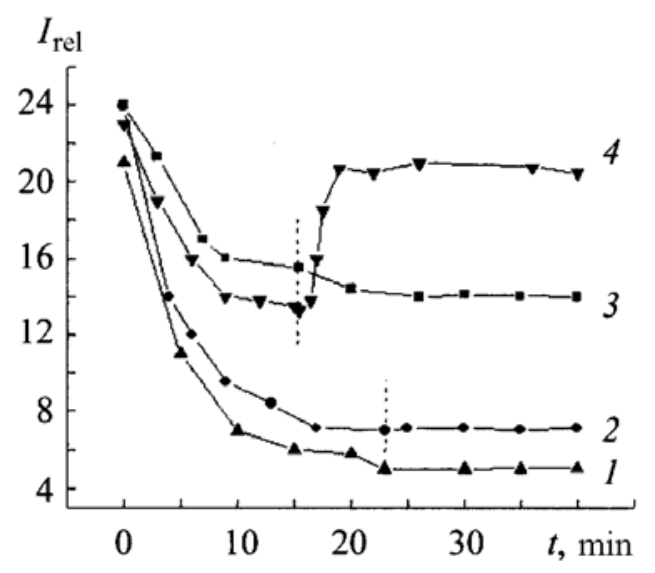

Fig. 2. Time dependence of the relative intensity of luminescence for the dye in the tumor during and after exposure to light with exposure energy dose 280 $\mathrm{J} / \mathrm{cm}^{2}$ for power density $200(1,2)$ and $300(3,4) \mathrm{mW} / \mathrm{cm}^{2}$. Dashed line: time at which the exposure to light stopped.

With an increase in the power density of the light exposure up to $300 \mathrm{~mW} / \mathrm{cm}^{2}$ with the same exposure energy dose of $280 \mathrm{~J} / \mathrm{cm}^{2}$, in the irradiated section of the tumor we also detected a decrease in fluorescence intensity, an increase in the half-width of the dye spectrum by 1-3 nm, and a short-wavelength shift of the maximum by $1 \mathrm{~nm}$. For 2 hours after the end of light exposure, the signal and shape of the fluorescence spectrum in the irradiated section of the tumor in most cases remained unchanged, but for some samples recovery of the intensity and shape of the fluorescence spectrum occurred (Fig. 2, curve 4).

Increasing the power density up to $600 \mathrm{~mW} / \mathrm{cm}^{2}$ for a light dose of $280 \mathrm{~J} / \mathrm{cm}^{2}$ in the irradiated section of the tumor still led to a long-wavelength shift of the maximum in the fluorescence spectrum by $1-2 \mathrm{~nm}$. After the end of light exposure, we observed a rather fast (within $\sim 20$ minutes) recovery of the intensity and shape of the dye fluorescence spectrum until the original parameters were reached. In this case, the intensity of fluorescence and accordingly also the PD1 concentration undergo more significant changes at a lower power density for the light exposure $\left(200 \mathrm{~mW} / \mathrm{cm}^{2}\right.$ ) (Fig. 2). During exposure to light with power density $200 \mathrm{~mW} / \mathrm{cm}^{2}$, the dye concentration in the tumor decreased by $65 \%$; for a power density of $300 \mathrm{~mW} / \mathrm{cm}^{2}$, by $45 \%$; and for a power density of $600 \mathrm{~mW} / \mathrm{cm}^{2}$, the concentration decreased by no more than 20-30\%.

We analyzed the correlation between the extent of damage to tumor tissues observed as a result of light exposure and the spectral changes. We found that tumor necrosis down to a depth of $2 \mathrm{~cm}$ is accompanied by a shortwavelength shift of the fluorescence spectrum of PD1 and an increase in its half-width after the end of light exposure; the intensity of fluorescence of PD1 in the samples is not recovered to the original level. Light exposure using radiation with power density $200 \mathrm{~mW} / \mathrm{cm}^{2}$ led to tumor tissue necrosis down to a depth of $2 \mathrm{~cm}$ in each PCT session. On exposure to radiation with power density $300 \mathrm{~mW} / \mathrm{cm}^{2}$, the same depth of tumor tissue necrosis was detected for samples in which, after the end of light exposure, the signal and shape of the fluorescence spectrum in the irradiated section of the tumor remained unchanged, while at $600 \mathrm{~mW} / \mathrm{cm}^{2}$ the depth of tissue necrosis was no greater than $5 \mathrm{~mm}$.

A decrease in therapeutic efficiency of photo-induced damage with an increase in the power density of the light was noted in $[8,9]$ and interpreted as a consequence of a decline in the tissue oxygen concentration (induced tissue hypoxia). We saw this type of effect for PD1 in the HeLa cell culture. Thus increasing the power density from 10 to $25 \mathrm{~mW} / \mathrm{cm}^{2}$ while leaving unchanged the exposure energy dose of $10 \mathrm{~J} / \mathrm{cm}^{2}$ led to an increase by a factor of 1.4 in the fraction of living HeLa tumor cells.

The changes in the fluorescence spectra of the dye in tissues observed as a result of exposure to light may be due to a change in the optical properties of the biological tissues, the effects of a decrease in the dye concentration (re-absorption or the presence of associates) or luminescence of accumulated photoproducts. By varying the concentration of the dye injected into the rats within the range $1-5 \mathrm{mg} / \mathrm{kg}$, we checked for a possible effect from an internal 


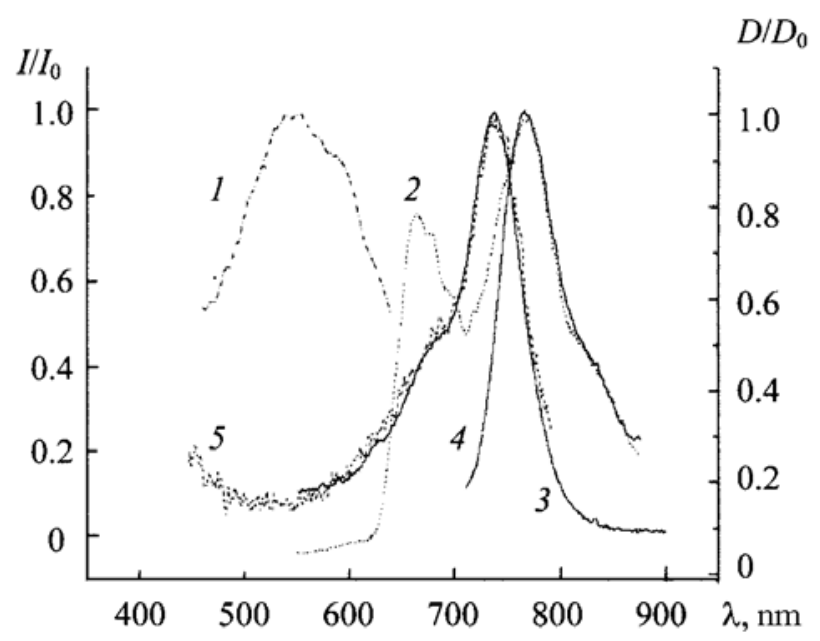

Fig. 3. Absorption spectrum of dye PD1 in HeLa cells (5), fluorescence spectra $(2,4)$ and fluorescence excitation spectra $(1,3)$ for PD1 in HeLa cells after exposure to light $\left(10 \mathrm{~J} / \mathrm{cm}^{2}, \lambda=740 \mathrm{~nm}, 10 \mathrm{~mW} / \mathrm{cm}^{2}\right) ; \lambda_{\text {ex }}=540 \mathrm{~nm}$ (2), $700 \mathrm{~nm}(4) ; \lambda_{\operatorname{det}}=663 \mathrm{~nm}(1), 810 \mathrm{~nm}(3)$.

filter or the presence of dye associates in the tumor. The intensity of the detected fluorescence signal for PD in vivo was proportional to the increase in the concentration, while the shape of the fluorescence spectra matched for all dye concentrations. These data suggest the absence of any concentration effects for the dye concentrations used.

The decrease in the fluorescence intensity of PD1 during exposure to light, which corresponds to a decrease in the dye concentration in the tumor tissue, is due to its photodegradation. With the aim of establishing the possible effect on the fluorescence spectrum of PD1 in the tissue from luminescence of photoproducts accumulated when treated with laser radiation, we studied the spectral properties of the dye in a model system: HeLa tumor cells. On illumination in the long-wavelength absorption band of the dye in HeLa with light from a semiconductor laser $(\lambda=$ $740 \mathrm{~nm}$ ), we observed an irreversible decrease in its concentration, which was apparent in the decrease in the fluorescence intensity of PD1 for $\lambda_{\mathrm{ex}}=683 \mathrm{~nm}$. At the same time, for excitation by light with $\lambda=500-550 \mathrm{~nm}$, the intensity of the luminescence band with maximum at $663 \mathrm{~nm}$ increased symbatically with the light exposure time (Fig. 3, curve 2). In the fluorescence excitation spectra of samples exposed to light with $\lambda_{\operatorname{det}}=663 \mathrm{~nm}$, there is a band with maximum at $545 \mathrm{~nm}$, while this band does not appear in the spectra of freshly prepared samples of the dye in HeLa. Consequently, the appearance of the band in the region of minimal absorption of the dye and fluorescence at $\lambda=$ $663 \mathrm{~nm}$ is due to accumulation of its photoproducts in the cells. Similar patterns for the change in spectral properties of polymethine dyes are observed on exposure to light in organic solvents [10]. For excitation of fluorescence with $\lambda=683 \mathrm{~nm}$, luminescence of photoproducts did not appear in the light-exposed samples. The shape and position of the fluorescence spectra of the dye in vitro (for excitation at $\lambda=683 \mathrm{~nm}$ before exposure to light and in its early stages for different power densities of the light) remained unchanged. The shape of the fluorescence excitation spectra for PD1 in HeLa cells after exposure to light for $\lambda_{\text {det }}=810 \mathrm{~nm}$ matched its absorption spectrum in unirradiated samples. Based on these data, we can conclude that the observed changes in the fluorescence spectrum of the dye in the model system upon exposure to light, and consequently also in vivo, are not due to the appearance of luminescence from photoproducts of PD1. In establishing the reasons for the changes in the shape and position of the spectrum, we must take into account the fact that the fluorescence of the dye PD1 in tissues in vivo can be affected by endogenous biomolecules that may have appreciable absorption in the same spectral region [11]. The fluorescence spectra of the studied PD1 overlap with absorption bands of hemoglobin, melanin, and water [12]. A change in the absorption spectra of water and melanin on exposure to light seems unlikely, but the ratio of the different forms of hemoglobin (oxyhemoglobin, deoxyhemoglobin, and methemoglobin) may change. We should take into account the fact that during the PCT session, due to the decrease in oxygen in the tumor tissue, the ratio of the different forms of hemoglobin 
changes: the oxyhemoglobin fraction decreases $[13,14]$. The absorption spectra for oxyhemoglobin, deoxyhemoglobin, and methemoglobin in the fluorescence band of PD1 $(710-875 \mathrm{~nm})$ have significant differences $[12,15,16]$, so a change in the ratio of the concentrations of the different forms of hemoglobin can have an effect on the shape of the fluorescence band of the dye. Accordingly, we carried out a numerical calculation of the PD1 spectra, taking into account absorption of some of the fluorescence radiation by different forms of hemoglobin.

In the spectrometric system used, fluorescence of the dye molecules in the tissues is excited and detected at a depth of $\sim 1.5 \mathrm{~cm}$ from the surface [17]. Therefore in the general case, for the fluorescence spectrum of PD1 in vivo, due to emission by the dye molecules for a biological tissue depth down to $l=1.5 \mathrm{~cm}$, taking into account absorption by different forms of hemoglobin according to the Bouguer-Lambert-Beer law, we can write:

$$
I(\lambda)=I_{0}(\lambda) \int_{0}^{1.5} 10^{l\left(C_{\mathrm{HbO}_{2}} \varepsilon_{\mathrm{HbO}_{2}}(\lambda)+C_{\mathrm{Hb}} \varepsilon_{\mathrm{Hb}}(\lambda)+C_{\mathrm{MeHb}} \varepsilon_{\mathrm{MeHH}}(\lambda)\right)} d l,
$$

where $I_{0}(\lambda)$ is the fluorescence spectrum emitted by the dye molecules from a depth $l ; C_{\mathrm{HbO}_{2}}, C_{\mathrm{Hb}}, C_{\mathrm{MetHb}}$ are the concentrations of oxyhemoglobin, deoxyhemoglobin, and methemoglobin respectively; $\varepsilon_{\mathrm{HbO}_{2}}(\lambda), \varepsilon_{\mathrm{Hb}}(\lambda), \varepsilon_{\mathrm{MetHb}}(\lambda)$ are the molar absorption coefficients of oxyhemoglobin, deoxyhemoglobin, and methemoglobin, which are taken from [12, 13, $15,16]$.

According to the data in [11], the scattering coefficient for biological tissues varies insignificantly in the spectral range corresponding to the fluorescence band of PD1, and cannot have a substantial effect on the position and shape of its spectrum. Accordingly, scattering was not considered in this model. In order to calculate the hemoglobin concentration in the rat tumor tissue, we took into account the total blood volume, the weight and size of the animal. The hemoglobin concentration in animal blood is $110-120 \mathrm{~g} / \mathrm{L}$ [18]. Because the signal was averaged over the volume of the samples by using light beams of diameter $\sim 5 \mathrm{~mm}$ on the surface, in the model we assumed that the blood was uniformly distributed over the considered volume. In this case, we took into account a correction for the weight and volume of bone tissue, which does not contain a large amount of blood.

Due to the change in the ratio of the different forms of hemoglobin after the end of the PCT session, the expression for the detected fluorescence spectrum of PD1 based on Eq. (1) can be rewritten as follows:

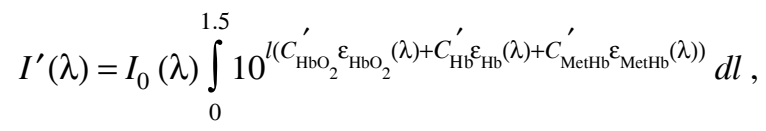

where $I^{\prime}(\lambda)$ is the calculated fluorescence spectrum of PD1 in vivo; $C_{x}^{\prime}$ are the concentrations of the different forms of hemoglobin after exposure to light.

By varying the ratio of the $\mathrm{Hb}, \mathrm{HbO}_{2}$, and MetHb concentrations according to formula (2), we did a numerical calculation of the fluorescence spectrum of the dye in vivo after the exposure to light, taking into account absorption by the blood components. In this case, the parameters characterizing the changes in the concentration of the different forms of hemoglobin as a result of exposure to light, $\Delta C=C-C_{x}^{\prime}$, and specifically $\Delta C_{\mathrm{MetHb}}, \Delta C_{\mathrm{Hb}}$, $\Delta C_{\mathrm{HbO}_{2}}$, were determined by minimizing the function $\xi\left(\Delta C_{\mathrm{MetHb}}, \Delta C_{\mathrm{Hb}}, \Delta C_{\mathrm{HbO}_{2}}\right)$ :

$$
\xi\left(\Delta C_{\mathrm{HbO}_{2}}, \Delta C_{\mathrm{Hb}}, \Delta C_{\mathrm{MetHb}}\right)=\sum_{\lambda}\left(I^{\prime}(\lambda)=I_{\exp }(\lambda)\right)^{2} .
$$

The function $\xi\left(\Delta C_{\mathrm{MetHb}}, \Delta C_{\mathrm{Hb}}, \Delta C_{\mathrm{HbO}_{2}}\right)$ represents the square of the difference between the experimental fluorescence spectrum of PD1 in vivo after exposure to light and the spectrum calculated from formula (2). We established that the best agreement between the experimental and calculated fluorescence spectra of the dye in the irradiated section of the tumor is observed for exposure to light with power density $200-300 \mathrm{~mW} / \mathrm{cm}^{2}$, due to the significant increase in the methemoglobin concentration in the tumor tissue (Fig. 4). In this case, after irradiation, the methemoglobin concentration in the tumor tissue increased to up to $50 \%$ of the total hemoglobin concentration in the blood; the oxyhemoglobin fraction was $\sim 30 \%$ and the deoxyhemoglobin fraction was $\sim 20 \%$. According to [13], under normal conditions, the methemoglobin concentration in tissue can lie within the range of $1-5 \%$ of the total hemoglobin concentration in the blood, while a content of more than $60 \%$ methemoglobin in animal or human blood is a critical threshold [14]. According to the data obtained, a local increase in the methemoglobin concentration is observed 


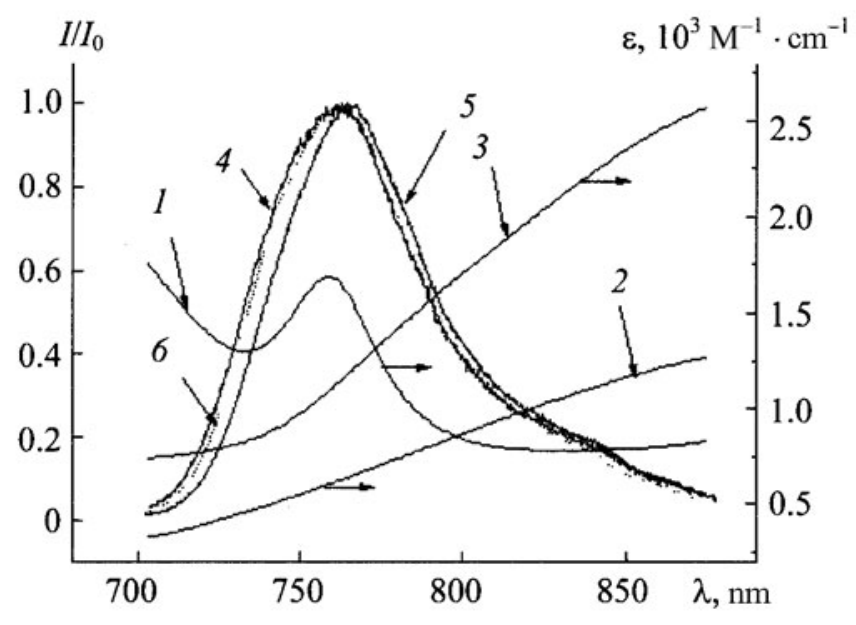

Fig. 4. Absorption spectra of deoxyhemoglobin (1), oxyhemoglobin (2), and methemoglobin (3); fluorescence spectra of dye PD1 in vivo after irradiation (4), before irradiation (5), and the calculated fluorescence spectrum of PD1 (6).

only in the irradiated section of the tumor tissue, which is damaged as a result of photochemical reactions. We should bear in mind that, according to the data in $[19,20]$, when blood components are exposed to laser radiation even in the absence of a photosensitizer, changes may occur in the hemoglobin structure: some of the oxyhemoglobin and deoxyhemoglobin are converted to methemoglobin.

For sections of the tumor exposed to light with power density $600 \mathrm{~mW} / \mathrm{cm}^{2}$, the best agreement between the experimental fluorescence spectrum of the dye and the calculated spectrum corresponds to a deoxyhemoglobin concentration of $\sim 60 \%$ of the total amount of hemoglobin. The results obtained (owing to the significant differences between the absorption spectra of the different forms of hemoglobin in the spectral region of emission of PD1) allow us to estimate the level of tissue oxygenation and the efficacy of the photochemotherapy from the changes in the detected fluorescence spectra.

Conclusion. Based on analysis of the spectral data for the dye PD1 in the near IR range, we can predict the depth and extent of tumor tissue necrosis achieved on exposure to light. We can assume that the main reason for the change in the fluorescence spectrum of PD1 as a result of photochemotherapy is a change in the ratio of the concentrations of the different forms of hemoglobin in the blood. By analysis of the spectral data in different stages of exposure to light, we can make changes in the protocol of the photochemotherapy session and improve its efficacy.

\section{REFERENCES}

1. M. B. Ericson, A.-M. Wennberg, and I. Larko, Therapeutics and Clinical Risk Management, 4, No. 1, 1-9 (2008).

2. T. J. Dougherty, C. J. Gomer, B. W. Henderson, G. Jori, D. Kessel, M. Korbelik, J. Moan, and Q. Peng, J. Natl. Cancer Inst., 90, No. 12, 889-905 (1998).

3. H. I. Pass, J. Natl. Cancer Inst., 85, 443-456 (1993).

4. E. F. Stranadko, Ros. Onkolog. Zh., No. 4, 52-56 (2000).

5. R. Richards-Kortum and E. Sevick-Muraca, Ann. Rev. Phys. Chem., 47, 555-606 (1996).

6. M. P. Samtsov, E. S. Voropai, D. G. Melnikau, L. S. Liashenka, A. A. Lugovskij, and Yu. P. Istomin, Zh. Prikl. Spektr., 77, No. 3, 438-444 (2010).

7. E. S. Voropay, ed., Spectral Instruments for Analytical Applications. Promising Developments [in Russian], Bel. Gos. Univ., Minsk (2005), pp. 5-9. 
8. T. Hasan and J. Parrish, in: J. F. Holland, E. I. Frei, R. J. C. Bast, D. W. Kufe, D. L. Morton, and R. R. Weisehselbaum, eds., Cancer Medicine, 4th Ed., William \& Wilkins, Baltimore (1996), pp. 739-751.

9. P. Vaupel, O. Thews, D. Kelleher, and M. Hoeckel, Strahlenther. Onkol., 174, No. 4, 6-12 (1998).

10. E. S. Voropay, A. P. Lugovskij, N. A. Pavlovskaya, and M. P. Samstov, Opt. Spektrosk., 70, No. 4, 819-825 (1991).

11. P. Taroni, A. Pifferi, A. Torricelli, D. Comelli, and R. Cubeddu, Photochem. Photobiol., 2, 124-129 (2003).

12. S. Matcher, C. Elwell, C. Cooper, M. Cope, and D. Delpy, Analyt. Biochem., 227, No. 1, 54-68 (1995).

13. O. Abdallah, W. Stork, K. Miller-Glaser, and A. Bolz, Biomedizinische Technik, 48 (2003).

14. S. A. Kutsenko, Principles of Toxicology [in Russian], Foliant, St. Petersburg (2004).

15. S. Matcher, M. Cope, and D. Delpy, Phys. Med. Biol., 39, 177-196 (1994).

16. W. Zijlstra, A. Buursma, and O. van Assendelft, Visible and Near Infrared Absorption Spectra of Human and Animal Haemoglobin, VSP, Utrecht (2000).

17. E. S. Voropay, M. P. Samtsov, V. N. Chalov, and É. A. Zhavrid, Zh. Prikl. Spektrosk., 68, No. 3, 359-362 (2001).

18. W. Hsing-Wen, E. M. Putt, M. J. Emanuele, D. B. Shin, E. Glatstein, and A. G. Yodh, Cancer Res., 64, No. 20, 7553-7561 (2004).

19. L. Randeberg, J. Bonesr?nning, J. Nelson, and L. Svaasand, Lasers Surg. Med., 34, No. 5, 414-419 (2004).

20. Y. Seto, M. Kataoka, and K. Tsuge, Forensic Sci. Int., 121, Nos. 1-2, 144-150 (2001). 